\title{
Power-to-Gas: Die Rolle der chemischen Speicherung in einem Energiesystem mit hohen Anteilen an erneuerbarer Energie
}

\author{
M. Lehner, P. Biegger, A. R. Medved
}

Die Umstellung der Energieversorgung auf erneuerbare Quellen (Wind, Photovoltaik) wird in Zukunft verstärkt die Volatilität in der Stromerzeugung erhöhen. Um eine ausgeglichene Leistungsbilanz im Stromnetz sicherzustellen, werden Speicher benötigt - nicht nur kurzzeitig, sondern auch saisonal. Die bidirektionale Kopplung bestehender Energieinfrastruktur mit dem Stromnetz kann hier Abhilfe schaffen, indem der Strom in Elektrolyseanlagen zur Wasserstofferzeugung genutzt wird. Der Wasserstoff kann Erdgas in der vorhandenen Infrastruktur (Gasspeicher, Pipelines) in begrenztem Umfang beigemischt werden oder in einer gaskatalytischen Reaktion, der Methanisierung, mit Kohlendioxid und/oder Kohlenmonoxid direkt zu Methan umgesetzt werden. Durch den Rückgriff auf die Erdgasinfrastruktur wird eine Entlastung der Stromnetze erreicht und eine Speicherung der erneuerbaren Energien auch über lange Zeiträume ermöglicht. Ein weiterer Vorteil dieser als „Power-to-Gas” bezeichneten Technologie ist, dass das so erzeugte Methan eine Senke für $\mathrm{CO}_{2}$-Emissionen darstellt, da damit fossile Quellen substituiert werden und $\mathrm{so}^{\mathrm{CO}_{2}}$ in einem geschlossenen Kreislauf geführt wird.

Die Forschung im Bereich der Power-to-Gas-Technologie adressiert derzeit technologische Fortschritte sowohl im Bereich der Elektrolyse als auch für die nachfolgende Methanisierung, insbesondere, um Investitionskosten zu senken. Im Bereich der Methanisierung sind lastflexible Verfahren zu entwickeln, die der fluktuierenden Wasserstoffbereitstellung angepasst sind. Die Wirtschaftlichkeit der Power-to-Gas-Prozesskette kann durch eine synergetische Einbindung in bestehende Industrieprozesse erhöht werden. Beispielsweise bietet ein integriertes Hüttenwerk ein vielversprechendes infrastrukturelles Umfeld, da zum einen kohlenstoffhaltige Prozessgase in großen Mengen anfallen, zum anderen der Sauerstoff als Nebenprodukt aus der Wasserelektrolyse einer direkten Nutzung zugeführt werden kann. Derartige Konzepte lassen einen wirtschaftlichen Einsatz der Power-to-Gas-Technologie in naher Zukunft erwarten.

Schlüsselwörter: Power-to-Gas; Methanisierung; erneuerbare Energie; Speicher

Power-to-Gas: the significance of chemical storage in an energy system containing high shares of renewable energy.

The restructuring of the energy supply towards renewable sources (wind, photovoltaics) will increase the volatility in power generation in future. In order to achieve a balance between supply and demand in the power grid, storage technologies are required, both short-term as well as long-term. The bidirectional link of the existing natural gas infrastructure with the power grid is enabled by the transformation of electric power in water electrolysis in a gaseous chemical energy carrier, hydrogen. Hydrogen can be admixed to the natural gas infrastructure (pipelines, gas reservoirs) in a limited amount, or can react with carbon dioxide and/or carbon monoxide in a catalytic reaction to methane. With the utilization of the natural gas infrastructure a release of the power grid as well as long-term energy storage are achieved. Another advantage of this "Power-to-Gas" technology is the use of $\mathrm{CO}_{2}$ emissions for the production of methane, and thus the reduction of greenhouse gas emissions by $\mathrm{CO}_{2}$-recycling and the substitution of fossil sources.

The actual research in the field of "Power-to-Gas" deals with technological progress both in electrolysis as well as methanation, particularly to decrease investment costs. In terms of methanation, load flexible processes have to be developed which are adjusted to the fluctuating hydrogen production. The economic viability of the Power-to-Gas process chain can be increased by a synergetic link to existing industrial production. For example, an integrated steel plant offers a promising infrastructure, since carbon containing process gases are available in huge amounts, and oxygen as byproduct of the water electrolysis can be used directly. Such concepts facilitate an economically advantageous application of the Power-to-Gas technology in near future.

Keywords: Power-to-Gas; methanation; renewable energy; storage

Eingegangen am 12. Jänner 2017, angenommen am 20. Februar 2017, online publiziert am 8. Mai 2017

(c) The Author(s) 2017. Dieser Artikel ist auf Springerlink.com mit Open Access verfügbar

\section{Einleitung und Problemstellung}

Eine der zentralen Herausforderungen bei der Umstellung der Stromerzeugung auf erneuerbare Energie ist deren räumlich dezentraler und zeitlich fluktuierender Anfall, insbesondere bei Windund Photovoltaikanlagen. Schon heute gibt es Zeiten, in denen das Angebot an erneuerbaren Strom nicht mehr im Stromnetz untergebracht werden kann, was zu negativen Strompreisen oder auch zur zeitweisen Abschaltung von Anlagen führt. Ein weiterer Ausbau der erneuerbaren Energieerzeugung ist ein erklärtes gesellschaftspoliti- sches Ziel [1], das für die EU im Jahr 2050 einen Anteil der erneu-

Lehner, Markus, Lehrstuhl für Verfahrenstechnik des industriellen Umweltschutzes, Montanuniversität Leoben, Franz Josef-Straße 18, 8700 Leoben, Österreich (E-Mail: markus.lehner@unileoben.ac.at); Biegger, Philipp, Lehrstuhl für Verfahrenstechnik des industriellen Umweltschutzes, Montanuniversität Leoben, Franz Josef-Straße 18, 8700 Leoben, Österreich; Medved, Ana Roza, Lehrstuhl für Verfahrenstechnik des industriellen Umweltschutzes, Montanuniversität Leoben, Franz Josef-Straße 18, 8700 Leoben, Österreich 


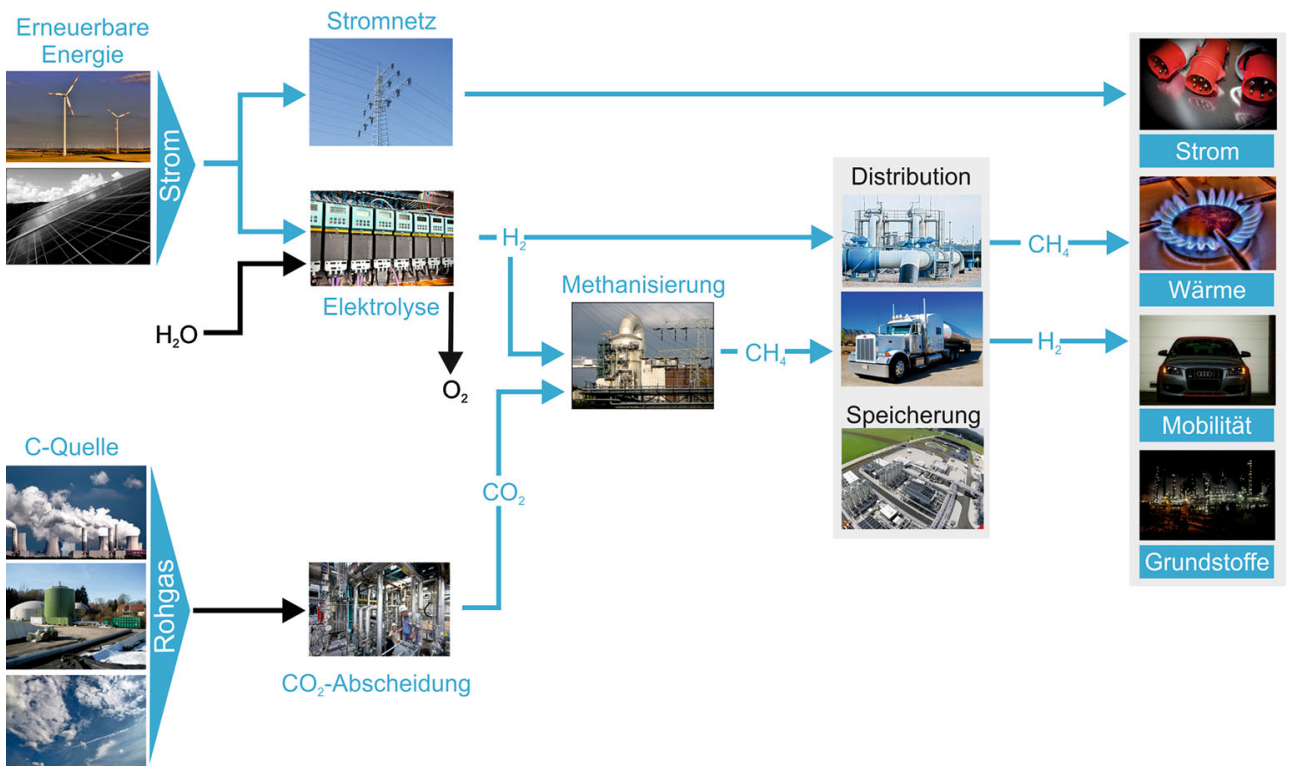

Abb. 1. Konzept der Power-to-Gas-Technologie

erbaren Energie am Bruttoendenergieverbrauch von $75 \%$ und am Stromverbrauch von $97 \%$ vorsieht. Daraus resultiert ein zukünftig stetig steigender Bedarf an Speicher für die erneuerbare Energie [2]. Die Speichertechnologien können kategorisiert werden in elektrische (z. B. Kondensatoren), elektrochemische (Batterien), chemische (z. B. Methan, Methanol), mechanische (z. B. Schwungrad, Druckluftspeicher) und thermische (z. B. Fernwärmespeicher, Latentwärmespeicher) Speicher [3]. Diese Speichertechnologien unterscheiden sich zum einen hinsichtlich ihrer Speicherdauer, ihrer volumetrischen Speicherdichte (Speicherkapazität) sowie bezüglich ihres technologischen Entwicklungsstandes [4]. Dabei ist der Bedarf an Speicher sowohl von der Geschwindigkeit des Ausbaus der erneuerbaren Energieerzeugung als auch von der Entwicklung anderer Flexibilisierungsoptionen, wie z. B. Lastmanagement oder Netzausbau, abhängig. Es ist aber offensichtlich, dass verschiedene Speichertypen, z. B. Kurz- und Langzeitspeicher, in Zukunft benötigt werden.

Chemische Speicher gehören zu den Langzeitspeichern. Die elektrische Energie wird dabei entweder in einer Wasserelektrolyse zur Herstellung von Wasserstoff, oder in einem Plasma bzw. Lichtbogen zur Synthese von C2-Verbindungen (Acetylen, Ethylen) aus beispielsweise Methan benutzt. Während Acetylen und Ethylen als chemische Grundstoffe zu Produkten weiterverarbeitet werden können, kann der Wasserstoff direkt als Energieträger verwendet werden. Es ist ein Einsatz in der Mobilität (z. B. wasserstoffgetriebene Fahrzeuge), zur Rückverstromung in Brennstoffzellen oder entsprechenden Gasturbinen, oder auch als wertvolles Reduktionsmittel in der chemischen, petrochemischen und metallurgischen Industrie möglich. Wasserstoff ist als chemischer Speicher jedoch nur bei einer entsprechend vorhandenen Infrastruktur sinnvoll, die lokal bereits verfügbar sein kann, flächendeckend aber erst aufgebaut werden muss. Die Beimengung von Wasserstoff zu Erdgas zur Nutzung des Erdgasnetzes als Speicherinfrastruktur ist limitiert [5]. Wasserstoff kann aber auch unter Einbeziehung einer Kohlenstoffquelle, wie z. B. $\mathrm{CO}_{2}$ und/oder $\mathrm{CO}$, in chemisch-katalytischen oder mikrobiologischen Prozessen zu Methan, Methanol oder anderen höheren Kohlenwasserstoffen (Fischer-Tropsch-Synthese) weiterverarbeitet werden. Die Route zu Wasserstoff oder Methan wird als
Power-to-Gas bezeichnet, die zu flüssigen Energieträgern als Powerto-Liquid.

In Abb. 1 ist die Power-to-Gas-Technologie in einem Überblick dargestellt [6]. Der wesentliche Vorteil bei der Umwandlung von erneuerbaren Strom in Methan liegt in der Möglichkeit der unbeschränkten Nutzung des vorhandenen Erdgasnetzes und der Erdgasspeicher sowie in der vielfältigen, technologisch vollständig entwickelten Verwendung von Methan respektive Erdgas als Energieträger für die Rückverstromung, zur Wärmeerzeugung, in der Mobilität oder auch als chemischer Grundstoff.

Die Forschungsaufgaben für die gesamte Power-to-Gas-Prozesskette liegen zum einen beim dynamischen Betriebsverhalten, insbesondere für die Methanisierung, der Wirtschaftlichkeit, dem (Gesamt-)Wirkungsgrad sowie der Bereitstellung einer geeigneten Kohlenstoffquelle für die Methanisierung [7]. Während der erste Prozessschritt, die Wasserelektrolyse, für den Fall der PEM (PolymerElektrolyt-Membran) Elektrolyse sehr dynamisch und somit auf ein schwankendes Stromdargebot flexibel reagierend betrieben werden kann, ist dies speziell für die katalytische Methanisierung in Festbettoder Wirbelschichtreaktoren nicht Stand der Technik [7]. Die Bereitstellung einer Kohlenstoffquelle kann die Wirtschaftlichkeit signifikant beeinflussen, daher ist ein besonderes Augenmerk auf den Standort mit dem spezifischen infrastrukturellen Umfeld für eine Power-to-Gas-Anlage zu legen. Mit diesen Anforderungen beschäftigen sich derzeit an der Montanuniversität Leoben laufende Forschungsaktivitäten, von denen ein Teil nachfolgend kurz vorgestellt wird.

\section{Lastflexible Methanisierung}

Bei der Methanisierung werden $\mathrm{H}_{2}$ und $\mathrm{CO}_{2}$ oder $\mathrm{CO} z u \mathrm{CH}_{4}$ und Wasserdampf umgewandelt. Diese Reaktionen laufen stark exotherm ab, höhere Drücke und niedrige Temperaturen verschieben die Gleichgewichtslage hin zu den Produkten. Temperaturabsenkung unter $200^{\circ} \mathrm{C}$ verlangsamt die Kinetik und führt zur unerwünschten Bildung von Nickeltetracarbonyl. Gängige katalytisch aktive Spezies sind Nickel-, Ruthenium- oder Eisen-Verbindungen [6].

$$
\begin{array}{ll}
\mathrm{CO}+3 \mathrm{H}_{2} \leftrightarrow \mathrm{CH}_{4}+\mathrm{H}_{2} \mathrm{O} & \Delta \mathrm{H}_{\mathrm{R}}^{0}=-206 \mathrm{~kJ} / \mathrm{mol} \\
\mathrm{CO}_{2}+4 \mathrm{H}_{2} \leftrightarrow \mathrm{CH}_{4}+2 \mathrm{H}_{2} \mathrm{O} & \Delta \mathrm{H}_{\mathrm{R}}^{0}=-164 \mathrm{~kJ} / \mathrm{mol}
\end{array}
$$



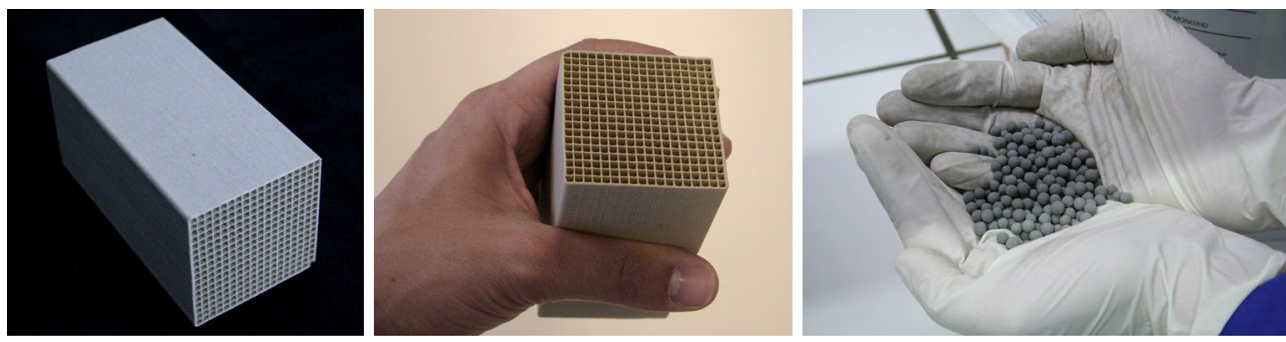

Abb. 2. Wabenkatalysator (links und Mitte), kommerzieller Schüttkatalysator (rechts)

Die Reaktionen finden üblicherweise in Festbett- oder Wirbelschichtreaktoren statt. Wirbelschichten ermöglichen eine nahezu isotherme Betriebsweise, da die entstehende Reaktionswärme aus dem Wirbelbett abgeführt werden kann, sind aber praktisch nicht lastflexibel. Zudem wird der verwirbelte Katalysator stark abrasiv belastet. In Festbettreaktoren müssen durch besondere Maßnahmen ein zu starker lokaler Anstieg der Temperatur unterbunden werden, wie z. B. durch Kreislaufführung der Gase oder durch Zwischenkühlung. Klassische Festbettreaktoren sind ebenfalls nur wenig lastflexibel.

Für die Anwendung der Methanisierung innerhalb der Power-toGas-Prozesskette ist ein Verfahren vorteilhaft, das in hohem MaBe lastflexibel ist, bei dem der Reaktor ganz oder teilweise im Stand-by gehalten werden kann und für das ein leichtes Scaleup mittels Modularisierung des Reaktors möglich ist. Diese Anforderungen resultieren aus dem fluktuierenden Stromdargebot, auf das insbesondere die PEM-Elektrolyse gut reagieren kann, die Kaltstartzeiten von einigen Sekunden bis Minuten aufweist und sehr dynamisch betrieben werden kann [7]. Zudem kann die Elektrolyseeinheit modular auf die gewünschte Nennleistung zusammengesetzt werden. In der Kopplung mit herkömmlichen Methanisierungsverfahren müssen sehr große Zwischenspeicher für den in der Elektrolyse produzierten Wasserstoff vorgesehen werden, um die katalytische Methanisierung gleichmäßig kontinuierlich betreiben zu können.

Im Zuge eines Research Studio Austria wurde an der Montanuniversität Leoben gemeinsam mit dem Industriepartner Christof Projects $\mathrm{GmbH}$ ein auf keramischen Wabenkatalysatoren beruhendes Methanisierungsverfahren entwickelt, das die genannten Anforderungen auf sich vereint [8]. Dabei dienen die keramischen Waben gleichzeitig als Katalysatoren und Wärmespeicher für die exotherme Reaktionswärme (Abb. 2). Als Material für die Waben eignet sich insbesondere Cordierit, das eine hohe Temperaturwechselbeständigkeit und ein hohes Wärmespeichervermögen aufweist.

Die keramischen Wabenkatalysatoren werden in einem Reaktor angeordnet, der in zwei oder mehr Kammern unterteilt ist, die gemeinsam oder auch nur einzeln angeströmt werden können (Abb. 3). Wird nur ein Teil der Kammern durchströmt, kann die Last sehr weit, z. B. bis auf $20 \%$ der Nennlast in Abhängigkeit der parallel vorhandenen Kammern, abgesenkt werden. Durch zyklisches Umschalten zwischen den Kammern bleiben alle Reaktorsegmente auf Reaktionstemperatur, da die exotherme Reaktionswärme in den Waben gespeichert wird. Die Waben können in mehreren Schichten übereinander angeordnet werden, zwischen denen gekühlt und oder Gase zugeführt oder entnommen werden können [9]. Die keramischen Waben wurden ausführlich getestet und erreichen nach kurzer Entwicklungszeit nahezu die Leistung herkömmlicher Katalysatoren [8].
Tab. 1. Zusammensetzung und Menge diverser Kuppelgase bei voestalpine Stahl, Linz [10]

\begin{tabular}{|c|c|c|c|}
\hline Spezies & Tiegelgas & Kokereigas & Gichtgas \\
\hline Max. Volumenstrom $\left[\mathrm{Nm}^{3} / \mathrm{h}\right]$ & 75.000 & 65.000 & 800.000 \\
\hline $\mathrm{CO}_{2}[\mathrm{Vol}-\%]$ & 19,6 & 1,2 & 21,9 \\
\hline CO [Vol-\%] & 52,0 & 5,8 & 25,8 \\
\hline $\mathrm{H}_{2}[\mathrm{Vol}-\%]$ & 0,3 & 66,2 & 5,9 \\
\hline $\mathrm{CH}_{4}[\mathrm{Vol}-\%]$ & - & 21,9 & 0,5 \\
\hline $\mathrm{N}_{2}[\mathrm{Vol}-\%]$ & 28,1 & $\sim 2,9^{\mathrm{a}}$ & $\sim 45,9$ \\
\hline $\mathrm{C}_{x} \mathrm{H}_{y}[\mathrm{Vol}-\%]$ & - & $\sim 2,0^{\mathrm{a}}$ & - \\
\hline $\mathrm{H}_{2} \mathrm{~S}$ & - & $4-12 \mathrm{~g} / \mathrm{Nm}^{3 \mathrm{a}}$ & $\sim 14 \mathrm{~g} / \mathrm{Nm}^{3 \mathrm{a}}$ \\
\hline $\mathrm{NH}_{3}$ & - & $6-8 \mathrm{~g} / \mathrm{Nm}^{3 \mathrm{a}}$ & - \\
\hline
\end{tabular}

aWerte aus [11]

\section{Power-to-Gas im integrierten Hüttenwerk}

In Europa erfolgt die primäre Stahlproduktion hauptsächlich über die Route eines sogenannten integrierten Hüttenwerkes. Die Bezeichnung dieser Route basiert auf der Verschaltung von mehreren Aggregaten bzw. Fertigungsstufen an einem Standort. Ein integriertes Hüttenwerk ist charakterisiert durch eine komplexe Stoff- und Energiestromcharakteristik. Es fallen energiereiche, $\mathrm{CO}-, \mathrm{CO}_{2}-$ und $\mathrm{H}_{2}$ haltige Gase, sogenannte Kuppelgase, aus unterschiedlichen Prozessen an. Dazu zählen beispielsweise Tiegelgas aus dem Stahlwerk, Kokereigas aus der Kokerei sowie Gichtgas aus dem Hochofen. Diese werden nach dem Stand der Technik nach einer Reinigung energetisch verwertet und sind charakterisiert durch unterschiedliche Zusammensetzungen, z. B. was den Gehalt an $\mathrm{CO}, \mathrm{CO}_{2}, \mathrm{H}_{2}$ und Stickstoff $\left(\mathrm{N}_{2}\right)$ angeht. Der C-bzw. $\mathrm{H}_{2}$-Anteil zeigt deutliche Unterschiede, wie aus Tab. 1 hervorgeht.

Eine signifikante Erhöhung der Energieeffizienz des Stahlwerksprozesses kann durch eine Kopplung der klassischen Stahlproduktion mit der Power-to-Gas-Technologie erreicht werden [12]. Das Potential der Effizienzsteigerung ist Gegenstand eines aktuell laufenden Forschungsprojektes. Dabei wird erneuerbarer Strom über eine Wasserelektrolyse in den Produktionsprozess eingebunden. $\mathrm{H}_{2}$ steht so aus der Elektrolyse zur Reduktion $\mathrm{CO}$ bzw. $\mathrm{CO}_{2}$ aus den Kuppelgasen zur Verfügung. In der Methanisierung entsteht als Produkt Methan, das sowohl innerhalb des integrierten Hüttenwerkes als auch für die Einspeisung in das Erdgasnetz verwendet werden kann. Sauerstoff aus der Elektrolyse kann hüttenwerksintern eingesetzt werden.

Im Zuge eines im Jahr 2017 beginnenden FFG-Projektes werden an der Montanuniversität Leoben gemeinsam mit der voestalpine Linz und Donawitz, der K1-MET GmbH sowie der TU Wien und dem Energieinstitut an der JKU Linz optimale Prozessketten und Verschaltungsvarianten für die Einbindung von Power-to-Gas in ein Stahlwerk entwickelt. Zielgröße der Optimierung ist die Reduktion der 

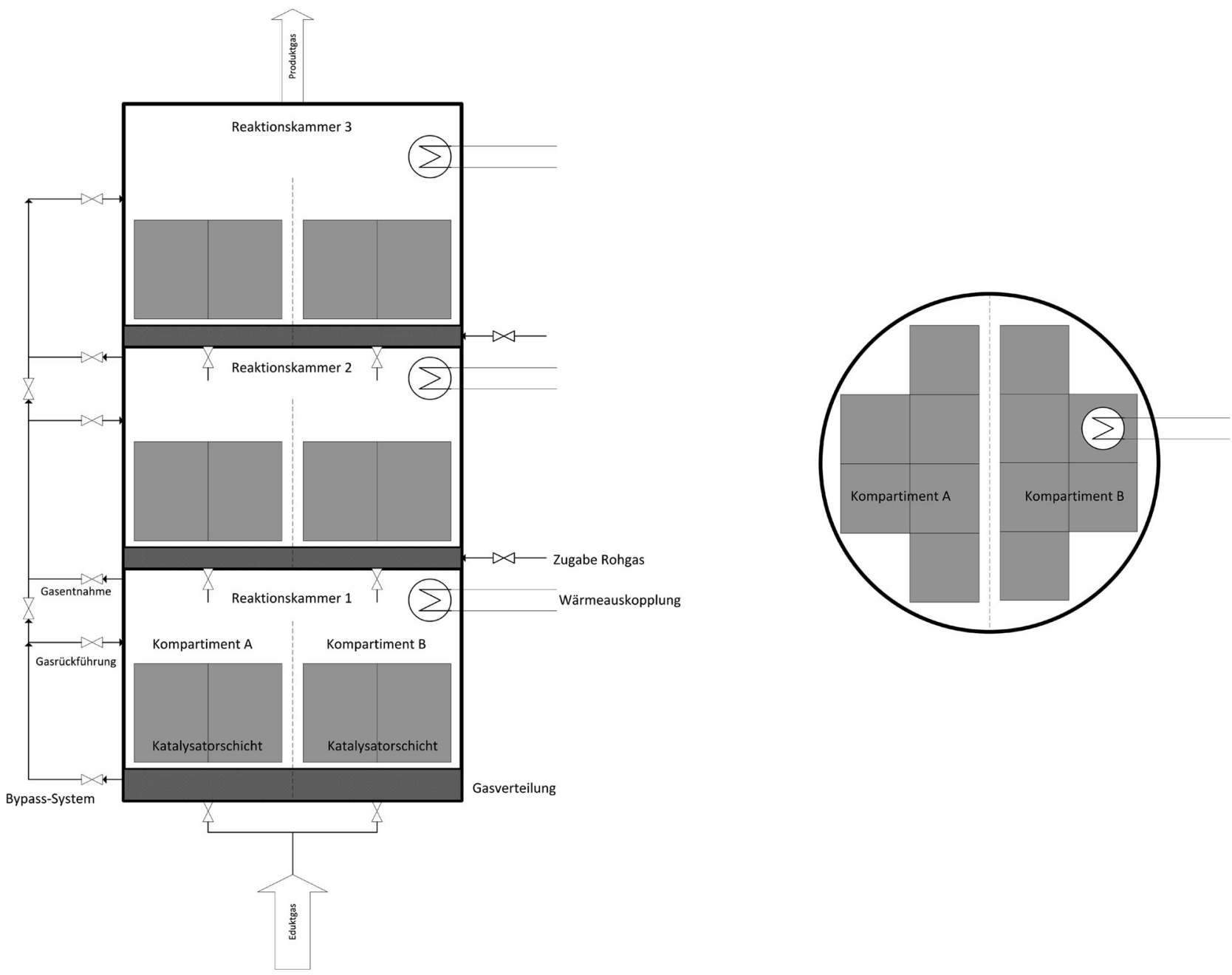

Abb. 3. Schema des lastflexiblen Methanisierungsverfahrens mit keramischen Wabenkatalysatoren

gesamten $\mathrm{CO}_{2}$-Emission des integrierten Hüttenwerkes unter Erhöhung der Energieeffizienz. Darüber hinaus wird der experimentelle Nachweis zu führen sein, dass sich Kuppelgase hinsichtlich ihrer Zusammensetzung und ihres mengenmäßigen Anfalls als Edukt für die Methanisierung eignen.

Abbildung 4 zeigt eine exemplarische Prinzipskizze, wie die Verschaltung zwischen einem integrierten Hüttenwerk und einer Power-to-Gas-Anlage zur Gewinnung eines methanreichen Produktgases aussehen kann. Die strichlierten Linien stellen diverse mögliche Produktverwertungswege dar. Die durchgezogenen Linien bedeuten Varianten der grundlegenden Stoffströme in der geplanten Prozessverkettung.

Aufgrund der Zusammensetzung kommen vor allem Tiegelgas und Kokereigas als mögliche Kuppelgasströme für eine Power-toGas-Umsetzung in Frage (Tab. 1). Im Konzept einer Verschaltung zwischen Hüttenwerk und Power-to-Gas-Anlage werden die Eduktgase vor dem Einsatz in der katalytischen Methanisierung einer Gasreinigung zugeführt, in der Verunreinigungen entfernt werden, beispielsweise Staub oder Schwefelverbindungen, welche vergiftend auf den Katalysator der Methanisierungsanlage wirken können. Vor allem Schwefelverbindungen wie $\mathrm{H}_{2} \mathrm{~S}$ (Gehalte siehe Tab. 1) sind als Katalysatorgift bekannt. Die Mengen an $\mathrm{H}_{2} \mathrm{~S}$ müssen auf jeden Fall genau und standortspezifisch bestimmt werden, um eine Deaktivierung des Katalysators zu verhindern. Eine Entstau- bung ist ebenfalls notwendig, da ein zu hoher Staubgehalt dazu führt, dass die aktive Katalysatoroberfläche belegt und so die katalytische Wirkung gehemmt wird. Zusätzlicher $\mathrm{H}_{2}$ wird durch eine Wasserelektrolyse zur Verfügung gestellt und so erneuerbare Energie in das integrierte Hüttenwerk eingespeist. Als Nebenprodukt fällt bei der Elektrolyse Sauerstoff an, welcher in das Hüttenwerk geleitet und dort verwendet werden kann. Beispielsweise könnte der Sauerstoff direkt in den Hochofen eingeblasen werden oder er wird der Luft beigemengt, welche im Winderhitzer aufgewärmt und anschließend als sogenannter Heißwind dem Hochofen zugeführt wird. Weitere Verwertungsmöglichkeiten für den Sauerstoff wäre die Verwendung im Stahlwerk zum Frischen des Roheisens. Damit ergibt sich eine weitere Effizienzsteigerung der Produktion, da auf die energetisch aufwändige Luftzerlegung verzichtet bzw. deren Größe reduziert werden kann. Der Wasserstoff aus der Elektrolyse tritt mit dem Kuppelgasgemisch in die Methanisierungsanlage ein. Das dort gebildete Methan-reiche Produktgas kann, nach einer optionalen Aufbereitung, in das Erdgasnetz eingespeist oder alternativ als Ersatz von fossilem Erdgas hüttenwerksintern verwendet werden. Es ist aber auch möglich, das Produktgas ohne weitere Aufreinigung hüttenwerksintern zu verwenden.

Aus diesem Beispiel wird deutlich, dass die Einbettung einer Power-to-Gas-Anlage in ein entsprechendes infrastrukturelles Um- 


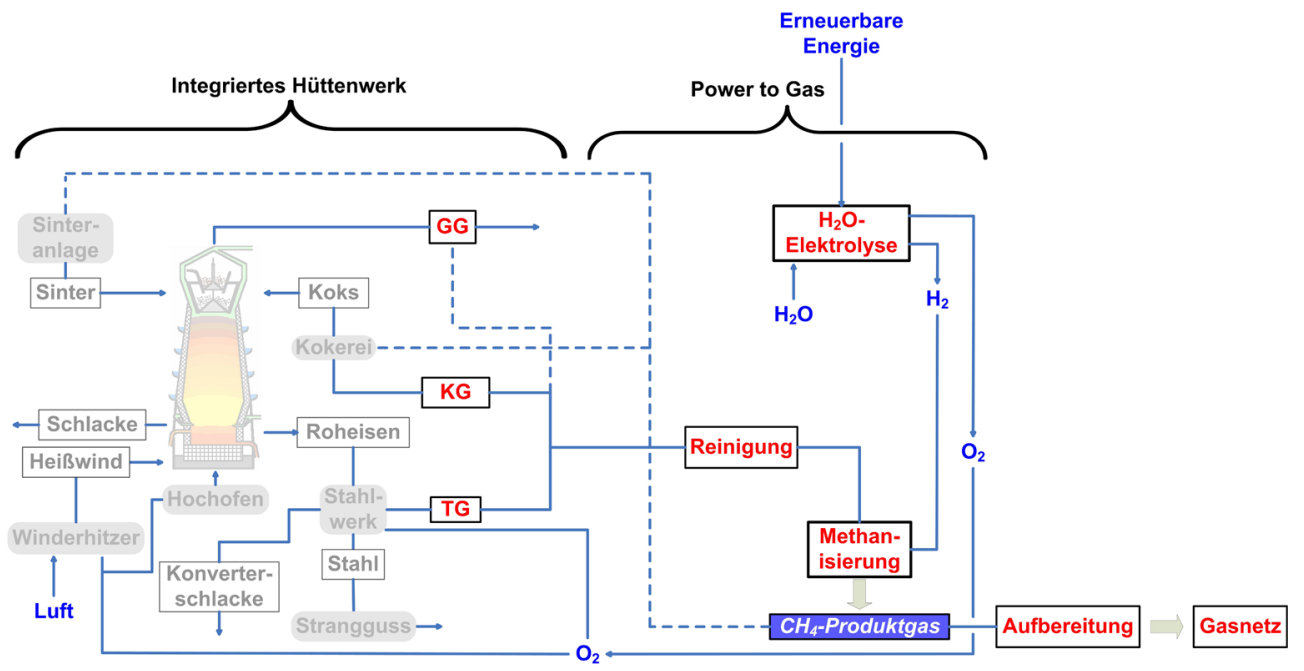

Abb. 4. Kopplung zwischen einem integrierten Hüttenwerk und dem Power-to-Gas-Konzept zur Methangewinnung. Abkürzungen: GG: Gichtgas; KG: Kokereigas; TG: Tiegelgas

feld signifikante Vorteile bietet, die die Gesamteffizienz, aber insbesondere auch die gesamte Wirtschaftlichkeit der chemischen Speicherung erneuerbarer Energie signifikant erhöhen kann. Die Entwicklung optimaler Verschaltungsvarianten sowie eine genaue Quantifizierung beispielsweise des $\mathrm{CO}_{2}$-Einsparpotentials ist Gegenstand aktueller Forschung.

\section{Schlussfolgerungen}

Der Ausbau erneuerbarer Energieerzeugung erfordert in zunehmenden Maße Speicheroptionen. Es ist dabei derzeit noch offen, in welchen Umfang, auf welcher Zeitachse und welche Speichertechnologien gebraucht werden. Es steht aber außer Zweifel, dass eine Energieversorgung mit hohen Anteilen aus Erneuerbaren nicht ohne Kurz- und Langzeitspeicher auskommen wird. Unter den Langzeitspeichern bietet Power-to-Gas die Möglichkeit, auf die gut ausgebaute Infrastruktur des Erdgasnetzes zurück zu greifen. So können Investitionen in Transport- und Speicherinfrastruktur vermieden sowie auf vollständig entwickelte Nutzungstechnologien aufgesetzt werden.

Innerhalb der Power-to-Gas-Prozesskette gilt es, sowohl Elektrolyse als auch katalytische Methanisierung dynamisch und lastflexibel betreiben zu können. Ein neu entwickeltes Methanisierungsverfahren nutzt keramische Waben als Wärmespeicher und Katalysator. Es wird so eine bisher nicht realisierbare Lastflexibilität der Methanisierung ermöglicht. Die Wirtschaftlichkeit der Power-toGas-Prozesskette kann durch Nutzung von Synergien mit einem optimalen infrastrukturellen Umfeld erhöht werden. Eine aussichtsreiche Möglichkeit stellen dafür integrierte Hüttenwerke dar, die zum einen über geeignete Kohlenstoffquellen für die Methanisierung, zum anderen über Nutzungsmöglichkeiten für den Sauerstoff als Nebenprodukt der Elektrolyse sowie den energiereichen Methanisierungsprodukten verfügen. Neben der Einbindung von erneuerbarer Energie in den Produktionsprozess sowie deren chemischer Speicherung entsteht ein großes Potential zur Senkung der $\mathrm{CO}_{2}$ Emissionen aus energieintensiver Industrieproduktion. Ähnliche Potentiale sind beispielsweise in der Zement- oder Feuerfestindustrie zu vermuten.

\section{Danksagung}

Open access funding provided by Montanuniversität Leoben.

Open Access This article is distributed under the terms of the Creative Commons Attribution 4.0 International License (http://creativecommons.org/ licenses/by/4.0/), which permits unrestricted use, distribution, and reproduction in any medium, provided you give appropriate credit to the original author(s) and the source, provide a link to the Creative Commons license, and indicate if changes were made.

\section{Literatur}

1. Energiefahrplan 2050 - Mitteilung der Kommission an das europäische Parlament, den Rat, den europäischen Wirtschafts- und Sozialausschuss und den Ausschuss der Regionen, 2011. http://www.europarl.europa.eu/meetdocs/2009_2014/ documents/com/com_com(2011)0885_/com_com(2011)0885_de.pdf.

2. Energieforschungszentrum Niedersachsen (Hrsg.) (2013): Studie Eignung von Speichertechnologien zum Erhalt der Systemsicherheit. Abschlussbericht, FA 43/12, Goslar.

3. Sterner, M., Stadler, I. (2014): Energiespeicher - Bedarf, Technologien, Integration. Berlin: Springer.

4. Bajohr, S., Götz, M., Graf, F., Ortloff, F. (2011): Speicherung von regenerativ erzeugter elektrischer Energie in der Erdgasinfrastruktur. In Gwf-Gas-Erdgas (S. 200-210).

5. Deutscher Verein des Gas- und Wasserfaches e.V. (Hrsg.) (2013): Entwicklung von modularen Konzepten zur Erzeugung, Speicherung und Einspeisung von Wasserstoff und Methan ins Erdgasnetz. Abschlussbericht DVGW Förderkennzeichen G1-07-10, Bonn.

6. Lehner, M., Tichler, R., Koppe, M., Steinmüller, H. (2014): Power-to-Gas: Technology and Business Models. Cham: Springer.

7. Götz, M., Lefebvre, J., Mörs, F., McDaniel Koch, A., Graf, F., Bajohr, S., Reimert, R., Kolb, T. (2016): Renewable Power-to-Gas: A technological and economic review. Renew. Energy, 85, S.1371-1390.

8. Biegger, P., Medved, A. R., Lehner, M., Ebner, H. M., Friedacher, A. (2016): Methanisierung im Umfeld von Power to Gas. In Kurzfassungsband zum 14. Symposium Energieinnovation, Graz (S. 463-464). Graz: Verlag der Technischen Universität.

9. Österreichische Patentanmeldung durch Christof Management $\mathrm{GmbH}$, Anlage zur katalytischen Methanisierung. Aktenzeichen A51029/2015. Anmeldetag: 01.12.2015.

10. Voestalpine Stahl GmbH (2015): In Energiekennzahlen Standort Linz - GJ 2015, Linz.

11. Best Available Techniques (BAT) Reference Document for Iron and Steel Production, 2013

12. Schöß, M. A., Redenius, A., Turek, T., Güttel, R. (2014): Chemische Speicherung regenerativer elektrischer Energie durch Methanisierung von Prozessgasen aus der Stahlindustrie. Chem. Ing. Tech., 86, 734-739. 
Autoren

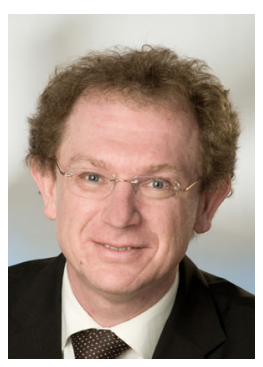

\section{Markus Lehner}

beendete das Studium der Verfahrenstechnik an der Technischen Universität München, Deutschland, 1992 und promovierte am Lehrstuhl für Thermodynamik der TU München im Jahr 1996. Er trat danach in das Anlagenbauunternehmen RVT Process Equipment $\mathrm{GmbH}$ in Steinwiesen, Bayern, ein und war dort zuletzt in leitender Funktion beschäftigt. Seit Oktober 2010 leitet er den Lehrstuhl für Verfahrenstechnik des industriellen Umweltschutzes an der Montanuniversität Leoben mit den Hauptarbeitsgebieten Gasreinigung und Energieverfahrenstechnik.

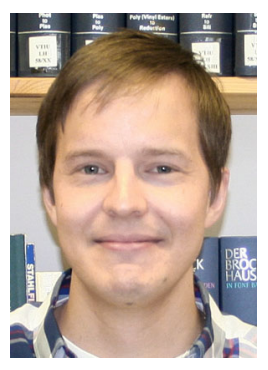

\section{Philipp Biegger}

studierte Industrielle Umweltschutz- und Verfahrenstechnik an der Montanuniversität Leoben, Österreich, und schloss das Studium 2011 mit der Masterprüfung ab. Seit 2012 beschäftigt er sich als wissenschaftlicher Mitarbeiter am Lehrstuhl für Verfahrenstechnik des industriellen Umweltschutzes der Montanuniversität Leoben mit dem Thema Powerto-Gas. Der Schwerpunkt liegt dabei auf der Weiterentwicklung des Methanisierungsverfahrens bzw. dessen Anwendungen in den verschiedensten Industriesektoren.

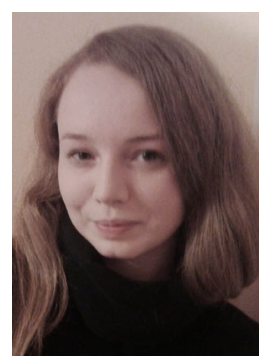

\section{Ana Roza Medved}

studierte Chemieingenieurwesen an der Fakultät für Chemie und Chemische Technologie der Universität Ljubljana, Slowenien, und erhielt 2016 das Diplom. Sie ist als wissenschaftliche Mitarbeiterin am Institut für Verfahrenstechnik des industriellen Umweltschutzes, Montanuniversität Leoben, im Bereich Power-to-Gas/Methanisierung beschäftigt. 